\title{
CORRESPONDENCE
}

\section{RECORDING PUPILLOGRAPH OF SIMPLE DESIGN}

\author{
To the Editorial Committee of the \\ British Journal of Ophthalmology
}

DEAR SIRs-After the publication of our paper in your October issue (British Journal of Ophthalmology, 35, 632) we found an article by J. Piltz (Neurol. Zbl., 1904, 23, 801, 853) describing an apparatus similar to that described by us.

It was of course impossible in 1904 to take full advantage of the idea; but we are eager to give the author the credit he deserves for his original conception at that early date.

\section{Yours faithfully;}

\section{H. VAN DER TWEEL.}

University Physical Laboratory,

AMSTERdam.

November 21, 1951.

\section{BOOK REVIEWS}

The Glaucomas. By H. S. Sugar. 1951. H. Kimpton, London. Pp. 469, 123 illus., bibl. (84s.)

It is a considerable time since a treatise on glaucoma has appeared and this book is welcome, if only in that it brings together and assesses the enormous amount of new observations which recent biochemical researches and new clinical techniques such as gonioscopy have produced. Sugar's work on glaucoma, first with the late Dr. Harry Gradle in Chicago, and then in his own clinic at Detroit, is, of course, well known, and he has become one of our contemporary authorities on gonioscopy and on the application and interpretation of the various provocative tests. The book is a small one, and does not enter into the subject exhaustively, but it gives a clear and readable outline of the whole clinical picture of the primary and secondary glaucomas as seen by one whose opinion is worth respect.

The adult primary glaucomas are classified as chronic simple glaucoma, which goes through a non-congestive and a congestive phase, and acute (narrow-angle) glaucoma, which also goes through a non-congestive and a congestive phase; it might be argued that the term " acute" may be slightly inappropriate to describe the many cases wherein minor rises of tension occur over many years without causing serious symptoms or inconveniencing the patient except for mild and transient attacks of halos and mistiness of vision.

The secondary glaucomas are discussed under various headings according as they are due to mechanical blockage of the trabecular spaces, to lack of a communication between the anterior and posterior chambers, to irritation of the ciliary body, to venous obstruction, to proliferating anastomotic vessels in the region of Schlemm's canal, to trauma, epidemic dropsy, angiomata, and, finally, to " mixed" and miscellanous causes. 
The book is very informative and futl of sound teaching, and gives a clear and wellintegrated idea of modern thought regarding this perplexing disease. If the reviewer were to be critical at all, he might suggest that the impression received is that the subject is over-simplified and that not enough stress is laid on the immensity of our ignorance regarding many of its fundamental problems. There are also one or two points with which every ophthalmologist might not agree-for example, that an important cause of primary glaucoma is osmotic failure in the functioning of the canal of Schlemm. Sugar accepts Friedenwald's view that this canal is supplied by arterioles which convey plasma into the canal and which in glaucoma become sclerosed, that the content of Schlemm's canal is plasma, and that the aqueous veins should therefore be called plasma veins. This theory is built on a single histological observation, the interpretation of which is somewhat problematical. Moreover, the suggestion is made in several places that glaucoma may be caused by over-secretion of the aqueous humour; again, there is very little evidence to support this view. There are one or two minor mistakes, such as the statement that acetylcholine is the substance responsible for the transmission of all nerve impulses across synapses when all "sympathetic" synapses are obviously meant. But, on the whole, no one interested in the problem of glaucoma will find it possible to overlook this excellent résumé.

Systemic Ophthalmology. By Arnold SorsBy. 1951. Butterworth, London. Pp. 712, 309 figs. (84s.).

This book represents the work of some thirty collaborators, each an expert in his or her own field. The designation of "systemic" is justified by Professor Sorsby in his short preface, where he points out that the adjective "medical" has now become too narrow for the general aspects of eye disease which include surgical, obstetric metabolic, and dermatological diseases and abnormalities, as well as other conditions Enormous though the subject of Systemic Ophthalmology has now become, its man aspects are here adequately covered.

The book is divided into six main sections dealing successively with:

(1) Prenatal pathogenic influences.

(2) Inflammation, allergic reactions, and infections.

(3) Nutritional, metabolic, and endocrine disturbances.

(4) Central nervous system.

(5) Cardiovascular and haemopoietic systems.

(6) Other general disturbances.

These sections are sub-divided into chapters, for each of which one of the collaborators is responsible ; it seems appropriate that the penultimate chapter should deal with senile changes in the eye and the last one with the eye at death. Systemic Ophthalmology thus gives a complete life history of what may happen to the eye, since the first chapter deals with concepts derived from experimental embryology.

One way of summing up one's reactions after reading this book is to adopt an Americanism and say that it constitutes a "must" so far as ophthalmic surgeons are concerned, and particularly for those who have to examine or lecture to students. Many examples could be quoted to show this, but two must suffice. The first concerns maternal rubella contracted during the first 4 months of pregnancy, which can produce, we are told, not only cataract in the offspring, but also buphthalmos and pigmentary retinal change, in addition to various systemic disturbances such as deaf-mutism, microcephaly, mental retardation, dental anomalies, and heart disease, the chances in favour of these changes occurring being $3: 1$. The second example concerns allergy; we all have some general conception of this condition, but the whole matter is clarified when it is divided into five main groups, and emphasis placed upon the difference between 